\title{
Low Voltage Imaging of Carbon Nanotubes With Tin-Palladium Particles Using STEM Detector in a FE-SEM.
}

\author{
C. Probst, R. Gauvin, R. A. L. Drew
}

Department of Mining, Metals and Materials Engineering, McGill University, M. H. Wong Bldg, 3610 University Street, Montreal, H3A 2B2, Quebec Canada.

The Field Emission Scanning Electron Microscope equipped with a STEM detector is a new device allowing new imaging possibilities at high spatial resolution. This paper intends to show the possibility of imaging carbon nanotubes using this device. In addition to imaging, it is also possible to estimate the resolution using the SMART software [1].

The microscope used in this work is an Hitachi S-4700 FE-SEM upgraded with a STEM detector. The multiwall carbon nanotubes were purchased from Nanostructured and Amorphous Materials Inc.. There were oxidized by refluxing 6 hours in a mixture of strong acids, followed by an immersion in an acidic solution of $\mathrm{SnCl}_{2}$ and $\mathrm{PdCl}_{2}$ and ultrasounded for 15 minutes, in order to deposit some tin-palladium particles.

The carbon nanotubes with tin-palladium were imaged using the different modes of FE-SEM upgraded with STEM detector. These modes of imaging were used: secondary electrons with the upper detector (SE), and transmitted electrons with the STEM detector in the bright field (TE) and dark field (DF) modes, as shown in Figure 1.

In Figure 1.A., SE mode was used. It is possible to see the particles of palladium-tin as small as $5 \mathrm{~nm}$ on the surface of the carbon nanotubes. Figure 1.B. shows the image obtain using the transmitted electron detector in bright field configuration. In this picture, the different contrast of the nanotubes compared to the particles is clearly seen. It is also possible to observe the interior of the tubes. Figure 1.C. shows a picture taken using the dark field mode. This image is noisier, so the resolution is degraded. Picture 1.D. was taken at the highest magnification possible with this microscope, 500k, using transmitted electrons in the bright field mode.

Figure 2. shows the evolution of the resolution calculated with SMART as a function of the magnification for the different imaging modes. Secondary electrons imaging and transmission electrons in bright field mode imaging have approximately the same estimated resolution and they are function of magnification, and the minimum resolution is about $2 \mathrm{~nm}$ for a magnification of $500 \mathrm{k}$. For the dark field mode, the calculated resolution is always poorer than the two other modes, around $5 \mathrm{~nm}$ at a magnification of 500k.

\section{References}

[1] D. C. Joy, J. Microsc. 208 (2002) 24.

Acknowledgements:

We would like to acknowledge NSERC for funding this work. 


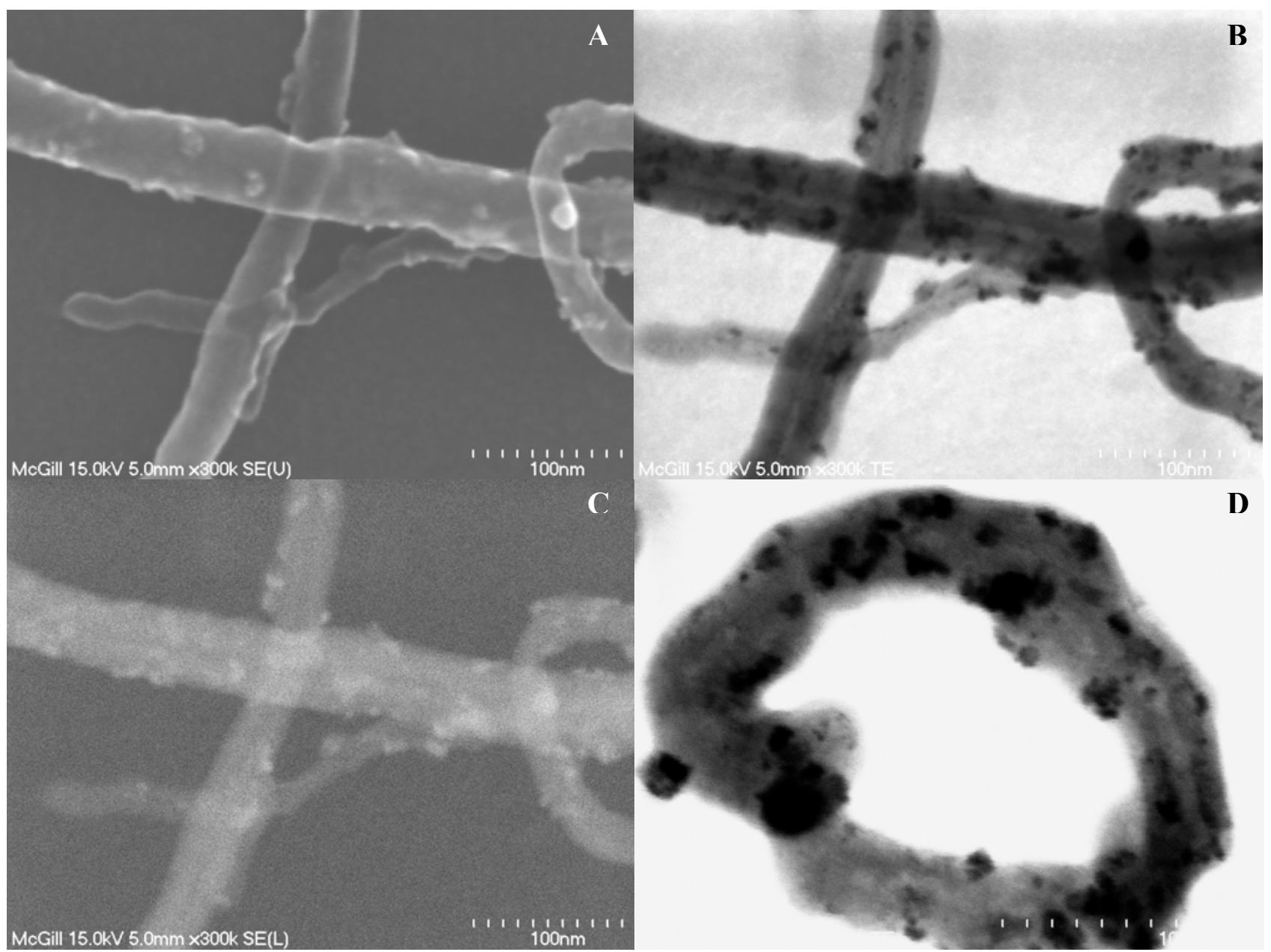

Fig. 1. Images of carbon nanotubes with tin-palladium particles using the different image modes of a FE-SEM upgraded with a STEM detector. A: SE mode. B: TE mode. C: DF mode. D: TE mode at high magnification. All pictures were taken at $5 \mathrm{~mm}$ with a voltage of $15 \mathrm{kV}$ and a magnification of $300 \mathrm{k}$, except for picture D where the magnification was $500 \mathrm{k}$.

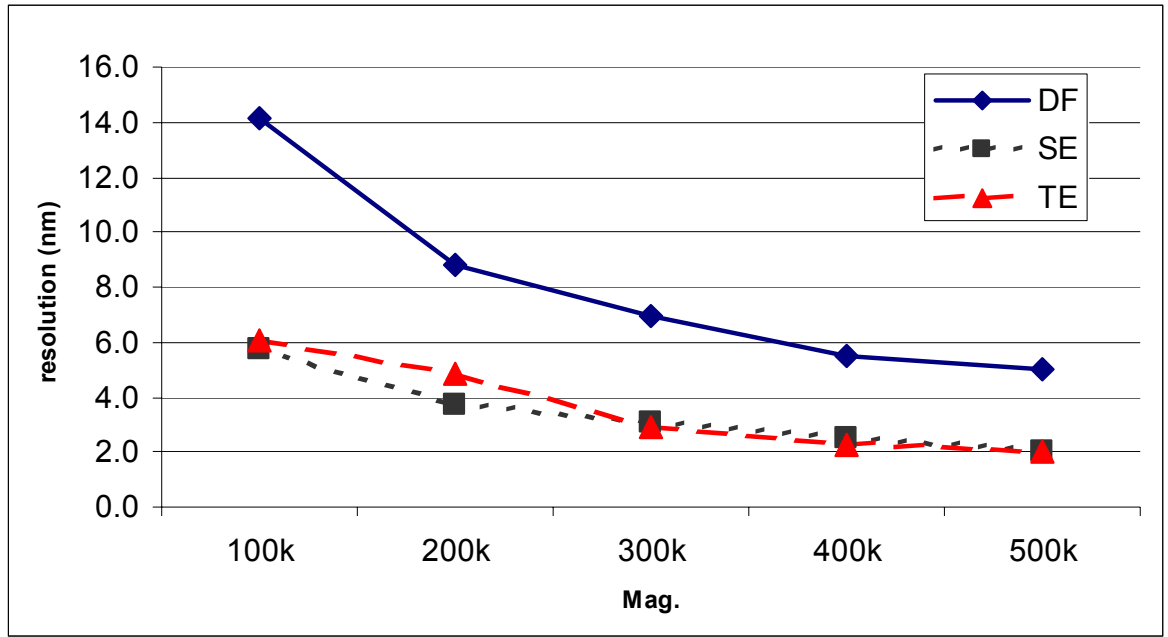

Fig. 2. Calculated resolution using SMART for the different modes of imaging at different magnifications $(\mathrm{V}=15 \mathrm{kV}, \mathrm{WD}=5 \mathrm{~mm})$. 